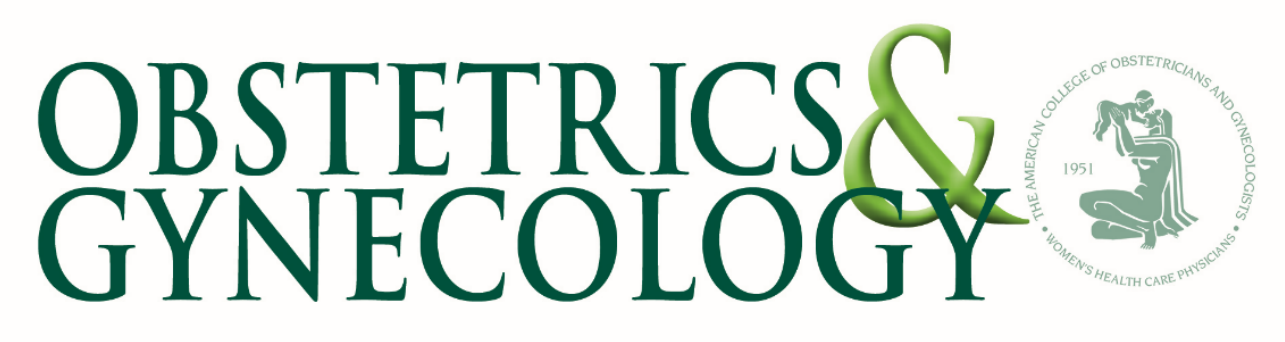

NOTICE: This document contains correspondence generated during peer review and subsequent revisions but before transmittal to production for composition and copyediting:

- Comments from the reviewers and editors (email to author requesting revisions)

- Response from the author (cover letter submitted with revised manuscript)*

- Email correspondence between the editorial office and the authors*

*The corresponding author has opted to make this information publicly available.

Personal or nonessential information may be redacted at the editor's discretion.

Questions about these materials may be directed to the Obstetrics \& Gynecology editorial office:

obgyn@greenjournal.org. 


$\begin{array}{ll}\text { Date: } & \text { Jul 26, } 2018 \\ \text { To: } & \text { "Michele Renee Hacker" } \\ \text { From: } & \text { "The Green Journal" em@greenjournal.org } \\ \text { Subject: } & \text { Your Submission ONG-18-1248 }\end{array}$

RE: Manuscript Number ONG-18-1248

Quality of information available online for abortion self-referral

Dear Dr. Hacker:

Your manuscript has been reviewed by the Editorial Board and by special expert referees. Although it is judged not acceptable for publication in Obstetrics \& Gynecology in its present form, we would be willing to give further consideration to a revised version.

If you wish to consider revising your manuscript, you will first need to study carefully the enclosed reports submitted by the referees and editors. Each point raised requires a response, by either revising your manuscript or making a clear and convincing argument as to why no revision is needed. To facilitate our review, we prefer that the cover letter include the comments made by the reviewers and the editor followed by your response. The revised manuscript should indicate the position of all changes made. We suggest that you use the "track changes" feature in your word processing software to do so (rather than strikethrough or underline formatting).

Your paper will be maintained in active status for 21 days from the date of this letter. If we have not heard from you by Aug 16, 2018, we will assume you wish to withdraw the manuscript from further consideration.

\section{REVIEWER COMMENTS:}

\section{Reviewer \#1:}

The authors performed internet searches using the Google, Bing \& Yahoo search engines, focusing on abortion information available in the 25 most populous US cities and 43 state capitals not in the large city list. They wanted to assess the quality of information available to women seeking abortion services. Using several standardized search terms they assessed the first 10 web pages, first 5 map results (to gage distance of the provider from the city) and advertisements facilitating abortion referrals. From 612 searches ( 68 cities $\times 3$ search terms $\times 3$ engines) they observed most web pages, maps and advertisements met their criteria for facilitating abortion referral while < one third hindered referral; the rest were classified as neutral i.e. not facilitating-not hindering. Advertisements were overall the most likely to hinder selfreferral. Significant US regional differences were also observed.

1. This study is probably not reproducible and may not represent good science. It seems likely that the top web pages, maps \& ads change regularly. Thus, at best this report provides a snapshot of information available in the 11 month interval 8/2016-6/2017. They should at least contact the search providers to assess the stability of this information. They should also learn and report how the order of web pages is determined. I used my city/state as an example with "abortion" and received 722,000 results in 0.6 seconds! Why did they examine only the first 10 webpages; how do they know this represents a legitimate sample? How well correlated were the results from the 3 engines? Exactly what method(s) was used to classify these search results? I was very concerned that they "anonymized" their searches, which means that their search results could have been very different had they initiated the search from the city under investigation.

2. They cite appropriate literature on the problems women face regarding provision of abortion services in America, but it is not clear that this report makes a substantial contribution to the literature. How can the practicing physician use this data? Can they speculate or suggest how this information might be used to change policy? Who is their best target audience?

3. They mention "quality" in the title $\&$ abstract objective/conclusion but never explicitly define that term for the reader.

4. Abortion services $>50$ miles from the city under investigation were classified as self-referral neutral. Can they justify this decision?

5. Line 157: they classified 5,800 web page results. Shouldn't that be $6,800(10 \times 680)$ ? 
Reviewer \#2:

This is a timely cross sectional study examining the quality of abortion self-referral information on the internet. It is imperative that providers and patients understand this especially today given the current anti-choice and access restrictive climate.

I feel like there needs to be more clarity on the difference between a webpage result and a location result.

Similarly, I don't understand how you have 612 searches if you based your searches on most populous cities and state capitals which does not equal 612.

Can you please explain the following - "the number of searches was based on the resources available for the project." I would probably delete as it makes it seem like you would do a better study if you had more resources.

When you performed your subanalysis based on distance to a publicly listed abortion provider, how did you find the "publicly listed abortion provider", also in a search?

In the interest of shortening your manuscript and making it more readable, you could present the results of all three categories simultaneously as opposed to each one separately which is very repetitive. For example, the percentage of webpages, locations, and ads that facilitated, hindered, or did not facilitate were \%, \%, and \% respectively. Each category revealed that distance from the nearest abortion provider and the Midwest predicted hindrance.

Can we unmask the search engines for the manuscript?

I would mention the recent supreme court decision re: pregnancy centers in California that do not have to provide information about abortion.

This manuscript is important for providers and patients alike and I would like to see something very practical in the Discussion section ie Patients will get the best information by using this engine to search this question and avoid ads. This is particularly important in the Midwest.

\section{Reviewer \#3:}

On first review this is a simple well done well written study about finding access to health services. However, more consideration finds that it has roots in the most vexing issues of modern society and we must take care in its interpretation. The study covers two issues abortion and modern communications. Abortion is not only grounded in health and medicine but also is at the center of debates in religion, ethics, economics, and of course politics. Communications and the internet are fundamental to education about and provision of health and medicine and also engulf any and all aspects of America today.

The simple thesis of the study is that women wanting to procure abortion services will go to the web to find such a provider or in other words, how and where can I get an abortion. The study searched web pages for advertisements, page results, and locations of abortion providers. The results of the searches are given in three tables and show a decided percentage mix of pro, uncertain, and anti-abortion information from web searches. The percentages of the three kinds of results for each of the three web types of web search approximate Gallop survey results about abortion of the past 40 years.

The authors conclude, "Individuals who use the internet to search for abortion services are at risk of encountering misinformation and of seeking care at centers that do not provide abortion care."

But their conclusion begs the question of the study. They assume from the outset, as their title implies, that those using the web to search for abortion providers will be exclusively women who want an abortion and thereby exclude all others who may use the web search technology to find such providers and information about abortion for other reasons. The few studies cited on anti-abortion pregnancy centers are carefully chosen to dismiss any conclusions aside from those of "misinformation". The investigators are fairly open about their pro-choice bias and perhaps should state it directly for clarity and transparency; this is after all supposed to be science rather than opinion.

Moreover and again rather than authors' opinion, there are advanced scientific and objective ways to critique the web. Connaway et al suggests that " . . . convenience is a critical factor. . ." in searching and Devine et al have listed extensive criteria to assess the quality of Health Websites.

In summary this work will be of use to both pro-choice and pro-life advocates in providing a sense of what information is available about abortion providers on the web although from a decided pro-choice perspective.

Connaway LS, Dickey TJ, Radford ML. "If it is too inconvenient I'm not going after it: " Convenience as a critical factor in 
information-seeking behaviors. Library \& Information Science Research 33 (2011) 179- 190

Devine T, Broderick J, Harris LM, Wu H, Hilfiker SW. Making Quality Health Websites a National Public Health Priority: Towards Quality Standards. Journal of Medical Internet Research. 18:8 (2016)

Gallop https://news.gallup.com/poll/1576/abortion.aspx accessed 6/29/18

\section{Reviewer \#4:}

This is a cross-sectional study of internet searches. While there are inherent limitations in a study using the internet, the authors acknowledge such limitations and despite these, this manuscript adds to the abortion literature and merits publication in a journal with a large general Ob/Gyn readership. While beyond the scope of this manuscript, the reader is left wondering 'how does this affect my practice?' or 'What do I do?'.

\section{Abstract:}

1. Lines 60-2: '43 cities not already included' confusing in the abstract, but makes sense once reading the manuscript. 2. Line 74: consider changing 'location results' to 'map results' (confusing as methods clearly states webpages, maps and ads, then change 'maps' to 'location').

Introduction: well written, clearly justifies need for study.

Materials and Methods: clear and concise.

3. It is not explained how the authors determined the 'truth' of abortion provider locations. How were abortion providers defined and identified prior to performing the web searches? What was done if the state of abortion provision changed during the study time period (i.e. during the time period a city lost a provider)?

Results:

4. The comparison between search engines is somewhat distracting and confusing. If it is significantly different in a consistent pattern, that is interesting. But it is hard to keep remembering facilitate versus not facilitate with anonymous different letters for the search engines. The authors may consider simplifying and removing the details of the differences between search engines and summarize as with the lines 217-221 in the discussion.

5. It would be interesting to know if distance to a provider is also associated with region. One wonders if distance to a provider and region are really just confounding variables.

\section{Discussion:}

6. Lines 221-25: Again, I wonder if distance to a provider and region are related.

7. Lines 243-259: while the anger is justified, this paragraph could be condensed with a focus on the internet aspects

(could consider deleting the sentences on lines 246-7, 250-2).

8. Lines 257: spell out NARAL

9. Lines 261-69: Adequately acknowledges the limitations of an internet study.

\section{STATISTICAL EDITOR COMMENTS:}

The Statistical Editor makes the following points that need to be addressed:

lines 123-124: Why were not more cities with smaller populations included? This strategy would seem to bias against more rural settings.

lines 159-210: While the figures are helpful, need to concisely state the important statistical comparisons in a Table format, so that the interested reader can see the differences in that format. Alternatively, could put the material in Figures in Table format with statistical comparisons and place that information as on-line supplemental.

Should repeat in legend figures the 3 search engines used.

\section{EDITOR COMMENTS:}

1. Thank you for your submission to Obstetrics \& Gynecology. In addition to the comments from the reviewers above, you are being sent a notated PDF that contains the Editor's specific comments. Please review and consider the comments in this file prior to submitting your revised manuscript. These comments should be included in your point-by-point response cover letter. 
McDermott and she will send it by email - kmcdermott@greenjournal.org.***

2. The Editors of Obstetrics \& Gynecology are seeking to increase transparency around its peer-review process, in line with efforts to do so in international biomedical peer review publishing. If your article is accepted, we will be posting this revision letter as supplemental digital content to the published article online. Additionally, unless you choose to opt out, we will also be including your point-by-point response to the revision letter, as well as subsequent author queries. If you opt out of including your response, only the revision letter will be posted. Please reply to this letter with one of two responses:

1. OPT-IN: Yes, please publish my response letter and subsequent email correspondence related to author queries.

2. OPT-OUT: No, please do not publish my response letter and subsequent email correspondence related to author queries.

3. Based on the forms that have been submitted, Dr. Neo and Dr. Nippita have not met the criteria for authorship. They should resubmit revised author agreement forms if they filled these out erroneously the first time. All updated and missing forms should be uploaded with the revision in Editorial Manager.

4. Our journal requires that all evidence-based research submissions be accompanied by a transparency declaration statement from the manuscript's lead author. The statement is as follows: "The lead author* affirms that this manuscript is an honest, accurate, and transparent account of the study being reported; that no important aspects of the study have been omitted; and that any discrepancies from the study as planned (and, if relevant, registered) have been explained." *The manuscript's guarantor.

If you are the lead author, please include this statement in your cover letter. If the lead author is a different person, please ask him/her to submit the signed transparency declaration to you. This document may be uploaded with your submission in Editorial Manager.

5. All studies should follow the principles set forth in the Helsinki Declaration of 1975, as revised in 2013, and manuscripts should be approved by the necessary authority before submission. Applicable original research studies should be reviewed by an institutional review board (IRB) or ethics committee. This review should be documented in your cover letter as well in the Materials and Methods section, with an explanation if the study was considered exempt. If your research is based on a publicly available data set approved by your IRB for exemption, please provide documentation of this in your cover letter by submitting the URL of the IRB web site outlining the exempt data sets or a letter from a representative of the IRB. In addition, insert a sentence in the Materials and Methods section stating that the study was approved or exempt from approval. In all cases, the complete name of the IRB should be provided in the manuscript.

6. Standard obstetric and gynecology data definitions have been developed through the reVITALize initiative, which was convened by the American College of Obstetricians and Gynecologists and the members of the Women's Health Registry Alliance. Obstetrics \& Gynecology will be transitioning as much as possible to use of the reVITALize definitions, and we encourage authors to familiarize themselves with them. The obstetric data definitions are available at http://links. Iww.com /AOG/A515, and the gynecology data definitions are available at http://links.Iww.com/AOG/A935.

7. Because of space limitations, it is important that your revised manuscript adhere to the following length restrictions by manuscript type: Original Research reports should not exceed 22 typed, double-spaced pages (5,500 words). Stated page limits include all numbered pages in a manuscript (i.e., title page, précis, abstract, text, references, tables, boxes, figure legends, and appendixes).

Please limit your Introduction to 250 words and your Discussion to 750 words.

8. Specific rules govern the use of acknowledgments in the journal. Please edit your acknowledgments or provide more information in accordance with the following guidelines:

* All financial support of the study must be acknowledged.

* Any and all manuscript preparation assistance, including but not limited to topic development, data collection, analysis, writing, or editorial assistance, must be disclosed in the acknowledgments. Such acknowledgments must identify the entities that provided and paid for this assistance, whether directly or indirectly.

* All persons who contributed to the work reported in the manuscript, but not sufficiently to be authors, must be acknowledged. Written permission must be obtained from all individuals named in the acknowledgments, as readers may infer their endorsement of the data and conclusions. Please note that your signature on the journal's author agreement form verifies that permission has been obtained from all named persons.

* If all or part of the paper was presented at the Annual Clinical and Scientific Meeting of the American College of Obstetricians and Gynecologists or at any other organizational meeting, that presentation should be noted (include the exact dates and location of the meeting). 
9. The most common deficiency in revised manuscripts involves the abstract. Be sure there are no inconsistencies between the Abstract and the manuscript, and that the Abstract has a clear conclusion statement based on the results found in the paper. Make sure that the abstract does not contain information that does not appear in the body text. If you submit a revision, please check the abstract carefully.

In addition, the abstract length should follow journal guidelines. The word limits for different article types are as follows: Original Research articles, 300 words; Reviews, 300 words. Please provide a word count.

10. Only standard abbreviations and acronyms are allowed. A selected list is available online at http://edmgr.ovid.com /ong/accounts/abbreviations. pdf. Abbreviations and acronyms cannot be used in the title or précis. Abbreviations and acronyms must be spelled out the first time they are used in the abstract and again in the body of the manuscript.

11. The journal does not use the virgule symbol (/) in sentences with words. Please rephrase your text to avoid using "and/or," or similar constructions throughout the text. You may retain this symbol if you are using it to express data or a measurement.

12. Please review the journal's Table Checklist to make sure that your tables conform to journal style. The Table Checklist is available online here: http://edmgr.ovid.com/ong/accounts/table_checklist. pdf.

If you choose to revise your manuscript, please submit your revision via Editorial Manager for Obstetrics \& Gynecology at http://ong.editorialmanager.com. It is essential that your cover letter list point-by-point the changes made in response to each criticism. Also, please save and submit your manuscript in a word processing format such as Microsoft Word.

If you submit a revision, we will assume that it has been developed in consultation with your co-authors, that each author has given approval to the final form of the revision, and that the agreement form signed by each author and submitted with the initial version remains valid.

Again, your paper will be maintained in active status for 21 days from the date of this letter. If we have not heard from you by Aug 16, 2018, we will assume you wish to withdraw the manuscript from further consideration.

Sincerely,

The Editors of Obstetrics \& Gynecology

2017 IMPACT FACTOR: 4.982

2017 IMPACT FACTOR RANKING: 5 th out of 82 ob/gyn journals

If you would like your personal information to be removed from the database, please contact the publication office. 
Dear Dr. Chescheir:

Thank you for inviting us to revise our manuscript for publication consideration in Obstetrics and Gynecology. We appreciate the time you and the reviewers took to thoroughly review our manuscript. We have included below a point-by-point response to each comment raised, and we look forward to hearing from you regarding these revisions.

Sincerely,

Michele R. Hacker, ScD, MSPH on behalf of all coauthors

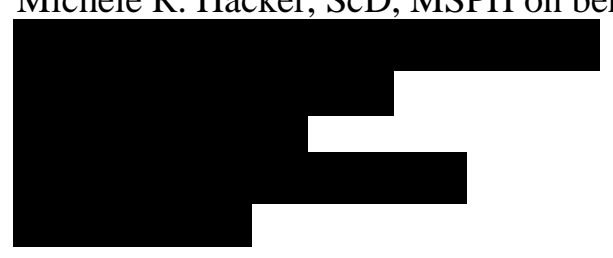

\section{REVEIWER \#1}

The authors performed internet searches using the Google, Bing \& Yahoo search engines, focusing on abortion information available in the 25 most populous US cities and 43 state capitals not in the large city list. They wanted to assess the quality of information available to women seeking abortion services. Using several standardized search terms they assessed the first 10 web pages, first 5 map results (to gage distance of the provider from the city) and advertisements facilitating abortion referrals. From 612 searches (68 cities $\times 3$ search terms $\times 3$ engines) they observed most web pages, maps and advertisements met their criteria for facilitating abortion referral while $<$ one third hindered referral; the rest were classified as neutral i.e. not facilitating-not hindering. Advertisements were overall the most likely to hinder self-referral. Significant US regional differences were also observed.

1. This study is probably not reproducible and may not represent good science. It seems likely that the top web pages, maps \& ads change regularly. Thus, at best this report provides a snapshot of information available in the 11 month interval 8/2016-6/2017. They should at least contact the search providers to assess the stability of this information. They should also learn and report how the order of web pages is determined. I used my city/state as an example with "abortion" and received 722,000 results in 0.6 seconds! Why did they examine only the first 10 webpages; how do they know this represents a legitimate sample? How well correlated were the results from the 3 engines? Exactly what method(s) was used to classify these search results? I was very concerned that they "anonymized" their searches, which means that their search results could have been very different had they initiated the search from the city under investigation.

The reviewer is correct that this is a cross-sectional study, and the top web pages, maps, and ads may change regularly. While the reviewer received 722,000 search results, the order of these results is far from random, as the search engines are incentivized to provide the searcher with the most relevant information. There was a great amount of overlap in the top ten search results across the three search engines, though the order may have differed slightly. We have added the following sentence to the paragraph in the Discussion describing the study limitations: 
Our study is also limited by its cross-sectional nature; while the top webpage results are expected to remain relatively stable, changes in the rankings of websites, map results, and ads do occur, and thus the results of this study represent a single snapshot in time.

Each search engine uses proprietary algorithms to rank websites that it sees as most relevant to the search terms. Ranking of the pages is determined by a combination of information from the webpage itself (e.g., content, title, keyword density) and information outside the webpage (e.g., number of links from other highly-ranked websites). We have modified the sentence describing this in the study limitations paragraph, and it now reads as follows:

However, because each search engine uses proprietary algorithms to rank websites for each search, the extent of this personalization is unknown.

We examined only the first ten webpages because that is typically what is shown on the first page of results, and nearly 92\% of traffic goes to the top ten results (Chitika, 2013). The last sentence of the second paragraph of the Methods now reads as follows:

We categorized up to the first 10 webpage results and up to the first five map results and ads, reflecting what is typically returned on the first page of results; we used only the first page of the search because previous work has shown that sites listed on the first page generate $92 \%$ of all traffic from an average search. ${ }^{18}$

We classified the results by hand. We added the following sentence to the third paragraph of the Methods:

If the website did not provide a clear indication of its proper categorization (e.g., abortion provider or crisis pregnancy center), one of the authors called the organization to determine whether they provided or referred for abortion services.

We agree with the reviewer that the search results could differ if we physically performed the searches within each city of interest as opposed to using an anonymous location, and this is acknowledged in the paragraph describing the study limitations. We initially attempted to use a virtual private network hosted within each city of interest to make it appear that each search was coming from within the city, but this was not feasible due to cost and availability of virtual private networks within each city. Thus, we chose to anonymize our location to prevent our actual location from influencing the search results. We tested this with a colleague in a different city who sent screenshots of her search results within her city, and they were nearly identical to the results obtained for that city using an anonymized location. We have added the following sentence to the limitations paragraph:

However, tests of searches within one city distant from the study location showed nearly identical results when searching within that city and when using an anonymized location at the study site. While use of a virtual private network would have further prevented our study location from being used in the search results, this was not feasible.

2. They cite appropriate literature on the problems women face regarding provision of abortion services in America, but it is not clear that this report makes a substantial contribution to the literature. How can the practicing physician use this data? Can they speculate or suggest how this information might be used to change policy? Who is their best target audience? 
We are using this information to collaborate with one of the major search engines to minimize the misleading information that is found online when searching for abortion providers. This information can also be used to raise awareness among clinicians about the importance of providers and staff being able to provide appropriate referrals given that patients are likely to encounter inaccurate information when searching online. Providers are already aware of this for other clinical issues, but it may be particularly problematic with regard to abortion care. We have revised the final paragraph of the Discussion as follows:

Although many searches resulted in referrals to abortion providers, some led to anti-abortion websites and crisis pregnancy centers. These proportions differed by search location, type of search result, and distance to an abortion provider. Because ads that hindered abortion selfreferral featured prominently in search results from all search engines, individuals who use the internet to locate abortion providers should be wary of information obtained through ads.

Patients who use the internet to locate abortion services are at risk of encountering misinformation and of mistakenly seeking care at facilities that do not provide the care they seek. As such, it is an ethical responsibility ${ }^{6}$ and critically important for health care providers and staff to be able to provide appropriate referrals for abortion care.

3. They mention "quality" in the title \& abstract objective/conclusion but never explicitly define that term for the reader.

We have added the following sentence to the third paragraph of the Methods:

Results that facilitated abortion referral were considered to be high quality, while results that hindered abortion referral were considered to be low quality.

4. Abortion services $>50$ miles from the city under investigation were classified as self-referral neutral. Can they justify this decision?

In cities that had abortion services located within 50 miles, abortion services located more than 50 miles away were considered neutral because closer options were viewed as more likely to result in the searcher obtaining services or a referral. These non-local abortion providers were often located hundreds or thousands of miles away from the city of interest. For cities that did not have abortion services located within 50 miles, the closest provider, regardless of distance, would have been considered "local" and thus would have been classified as facilitating self-referral.

5. Line 157: they classified 5,800 web page results. Shouldn't that be 6,800 (10 x 680)?

While all searches return hundreds of thousands of results, sometimes fewer than ten results are shown on the first page of the search results. In light of the data cited above showing how few people click to the second page of results, we chose to only classify the first page of results even if fewer than ten webpage results were shown. We have specified this in the Results as follows:

We categorized up to the first 10 webpage results and up to the first five map results and ads, reflecting what is typically returned on the first page of results; we used only the first page of the search because previous work has shown that sites listed on the first page generate $92 \%$ of all traffic from an average search. ${ }^{18}$ 


\section{REVEIWER \#2}

This is a timely cross sectional study examining the quality of abortion self-referral information on the internet. It is imperative that providers and patients understand this especially today given the current anti-choice and access restrictive climate.

6. I feel like there needs to be more clarity on the difference between a webpage result and a location result.

We have added information to the second paragraph of the Methods. The first sentence of this paragraph now reads as follows:

Web searches using the three search engines returned three categories of results-1) webpage results, which are the main search results and lead to webpages; 2) map results, which are shown as locations on an inset map; and 3) ads, which are shown at the top, side, and/or bottom of the page, around the webpage results.

7. Similarly, I don't understand how you have 612 searches if you based your searches on most populous cities and state capitals which does not equal 612.

We performed a total of 9 searches (3 search terms x 3 search engines) for 68 cities (25 most populous +43 state capitals that are not one of the prior 25 most populous cities); 9 x $68=612$. In order to make this easier for the reader to follow, we have modified the first sentence of the Results to read as follows:

We performed 612 searches using three search engines and three sets of search terms for 68 cities.

8. Can you please explain the following - "the number of searches was based on the resources available for the project." I would probably delete as it makes it seem like you would do a better study if you had more resources.

We based the number of cities on the time it took to classify the results by hand. We appreciate this comment and have removed that sentence from the manuscript and instead specified that this was a sample of convenience.

9. When you performed your subanalysis based on distance to a publicly listed abortion provider, how did you find the "publicly listed abortion provider", also in a search?

We used lists available from The National Abortion Federation and Planned Parenthood. In addition, we included facilities that were not listed by NAF or Planned Parenthood but that appeared in internet searches. We have modified this sentence in the Methods to now read as follows:

We performed sub-analyses based on distance of the location to a publicly-listed abortion provider; these providers were listed by the National Abortion Federation or Planned Parenthood or found on internet searches for abortion providers.

10. In the interest of shortening your manuscript and making it more readable, you could present the results of all three categories simultaneously as opposed to each one separately which is very repetitive. For example, the percentage of webpages, locations, and ads that facilitated, hindered, 
or did not facilitate were $\%, \%$, and \% respectively. Each category revealed that distance from the nearest abortion provider and the Midwest predicted hindrance.

We have removed the results comparing search engines from the text, which has shortened the length of the Results. While we believe that separating the results by search type is the clearest method of presentation, we will defer to the editor's preference for the order of results presentation.

11. Can we unmask the search engines for the manuscript?

We have elected to blind the search engines because our aim was more to get an overall sense of information available online for abortion self-referral as opposed to testing and comparing individual search engines. The webpage results were very similar between the search engines, and while search engine $C$ produced the best map results, it also had poor ad results, and thus we would be unable to make a single recommendation for the best search engine with which to selfrefer for abortion services. We removed comparisons between the search engines because this was not our aim and because it helped to streamline the text.

12. I would mention the recent Supreme Court decision re: pregnancy centers in California that do not have to provide information about abortion.

Thank you for this suggestion. After making revisions to the Discussion, we found that this addition seemed to flow best in the Introduction. We have added the following to the second paragraph of the Introduction:

Nearly one-third of surveyed obstetrics and gynecology and family medicine clinicians in Nebraska reported they would not provide a referral to abortion services, and 15\% reported they would instead give a referral either to a provider who did not offer abortions or to an adoption agency or crisis pregnancy center,$^{9}$ which is a facility that does not offer referrals for abortions; National Institute of Family and Life Advocates v. Becerra, decided in 2018 on free speech grounds that California could not compel these organizations to provide referrals for abortion services. ${ }^{10}$

13. This manuscript is important for providers and patients alike and I would like to see something very practical in the Discussion section i.e., patients will get the best information by using this engine to search this question and avoid ads. This is particularly important in the Midwest.

We have revised the last paragraph of the Discussion as follows:

Although many searches resulted in referrals to abortion providers, some led to anti-abortion websites and crisis pregnancy centers. These proportions differed by search location, type of search result, and distance to an abortion provider. Because ads that hindered abortion selfreferral featured prominently in search results from all search engines, individuals who use the internet to locate abortion providers should be wary of information obtained through ads.

Patients who use the internet to locate abortion services are at risk of encountering misinformation and of mistakenly seeking care at facilities that do not provide the care they seek. As such, it is an ethical responsibility ${ }^{6}$ and critically important for health care providers and staff to be able to provide appropriate referrals for abortion care. 


\section{REVIEWER \#3}

On first review this is a simple well done well written study about finding access to health services. However, more consideration finds that it has roots in the most vexing issues of modern society and we must take care in its interpretation. The study covers two issues abortion and modern communications. Abortion is not only grounded in health and medicine but also is at the center of debates in religion, ethics, economics, and of course politics. Communications and the internet are fundamental to education about and provision of health and medicine and also engulf any and all aspects of America today.

The simple thesis of the study is that women wanting to procure abortion services will go to the web to find such a provider or in other words, how and where can I get an abortion. The study searched web pages for advertisements, page results, and locations of abortion providers. The results of the searches are given in three tables and show a decided percentage mix of pro, uncertain, and anti-abortion information from web searches. The percentages of the three kinds of results for each of the three web types of web search approximate Gallop survey results about abortion of the past 40 years.

The authors conclude, "Individuals who use the internet to search for abortion services are at risk of encountering misinformation and of seeking care at centers that do not provide abortion care." But their conclusion begs the question of the study. They assume from the outset, as their title implies, that those using the web to search for abortion providers will be exclusively women who want an abortion and thereby exclude all others who may use the web search technology to find such providers and information about abortion for other reasons. The few studies cited on anti-abortion pregnancy centers are carefully chosen to dismiss any conclusions aside from those of "misinformation". The investigators are fairly open about their pro-choice bias and perhaps should state it directly for clarity and transparency; this is after all supposed to be science rather than opinion.

We agree with the reviewer that individuals who are not actively seeking abortion may search the internet for other information about abortion. However, the aim of our study was to determine how easy or difficult it is for individuals who are seeking abortion services to find information about these services online, and the search terms used in this study ("abortion” and city; "abortion provider” and city; "where to get an abortion" and city) were designed to reflect this. We believe that if individuals were seeking other information about abortion, they would use different and more specific search terms than those used in our study. For instance, we believe it is unlikely that individuals searching for general information about abortion would include the name of a city in their search, as was done for all searches in this study.

Our purpose of citing the studies on crisis pregnancy centers is to show that for people who are seeking abortion services, crisis pregnancy centers cannot provide them with the care they desire. While some individuals do receive wanted care from crisis pregnancy centers, this determination was not the aim of the study, and we have emphasized in several places (last paragraph of Introduction, first paragraph of Methods) that we assumed that individuals searching for abortion providers online were intending to locate abortion providers. We have revised the paragraph about crisis pregnancy centers to emphasize that the statements made are not opinion but rather supported by scientific studies. This paragraph now reads as follows:

Many results in our searches led to either crisis pregnancy centers or anti-abortion websites. Prior research has shown that crisis pregnancy centers intend to dissuade individuals from choosing abortion; they generally do not provide referrals for abortion care and are affiliated with anti-abortion organizations. ${ }^{16}$ One study of the websites of crisis pregnancy centers found that $80 \%$ provided incorrect information about abortion, ${ }^{17}$ and in our study, they were a common search result regardless of search terms or search engine. Another study reported that being unaware of a crisis pregnancy center's intended purpose can lead to surprise and anger about their refusal to provide or even refer for abortion 
care. ${ }^{13}$ Additionally, crisis pregnancy centers spend significant sums of money to advertise on internet search engines. ${ }^{16}$ Although Google banned such ads paid for by religious organizations in 2008, a settlement was reached, and the ads are now allowed. ${ }^{18}$ Google does have a policy against "misleading content," 19 and in 2014, Google removed the ads of many crisis pregnancy centers that were deemed to have misleading content. ${ }^{20}$ Despite this, we found that anti-abortion websites and crisis pregnancy centers were prominently featured among ads on all three search engines.

Moreover and again rather than authors' opinion, there are advanced scientific and objective ways to critique the web. Connaway et al suggests that " . . convenience is a critical factor. . ." in searching and Devine et al have listed extensive criteria to assess the quality of Health Websites.

We have cited the Connaway et al. study in the third paragraph of the Introduction. While Devine et al. have described criteria for evaluating the quality of health websites, the aim of our study was not to evaluate the full content of the websites identified, and thus these criteria are not applicable to our study.

In summary this work will be of use to both pro-choice and pro-life advocates in providing a sense of what information is available about abortion providers on the web although from a decided pro-choice perspective.

Connaway LS, Dickey TJ, Radford ML. "If it is too inconvenient I'm not going after it:" Convenience as a critical factor in information-seeking behaviors. Library \& Information Science Research 33 (2011) 179190

Devine T, Broderick J, Harris LM, Wu H, Hilfiker SW. Making Quality Health Websites a National Public Health Priority: Towards Quality Standards. Journal of Medical Internet Research. 18:8 (2016)

Gallop https://urldefense.proofpoint.com/v2/url?u=https-

3A news.gallup.com poll 1576 abortion.aspx\&d=DwIGaQ\&c=WknmpdNpvrlj2B5K1aWVqL1SOiF3 0547pqSuOmtwXTQ\&r=mkeq6YrJavXK4LFqUF5Xi3NvmT98swvGpbhzOVjfwG8\&m=qwhS5_sZYA9 mrs6wcTeiogt5iIejkfLPbMLfpVvCvMI\&s=HcKHFizR0lpx19L_ihZS2SNTkOcW-

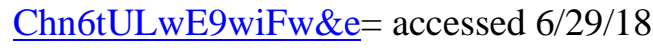

\section{REVIEWER \#4}

This is a cross-sectional study of internet searches. While there are inherent limitations in a study using the internet, the authors acknowledge such limitations and despite these, this manuscript adds to the abortion literature and merits publication in a journal with a large general Ob/Gyn readership. While beyond the scope of this manuscript, the reader is left wondering 'how does this affect my practice?' or 'What do I do?'

Abstract:

14. Lines 60-2: '43 cities not already included' confusing in the abstract, but makes sense once reading the manuscript.

We have modified this sentence to read as follows:

We used a standard protocol to perform internet searches for abortion services in each of the 25 most populous U.S. cities along with the 43 state capitals that are not one of the 25 most populous cities; searches were conducted from August 2016 to June 2017. 
15. Line 74: consider changing 'location results' to 'map results' (confusing as methods clearly states webpages, maps and ads, then change 'maps' to 'location').

We have ensured consistent use of the term "map results” throughout the manuscript.

16. Introduction: well written, clearly justifies need for study.

Thank you for this comment.

17. Materials and Methods: clear and concise.

Thank you for this comment.

18. It is not explained how the authors determined the 'truth' of abortion provider locations. How were abortion providers defined and identified prior to performing the web searches? What was done if the state of abortion provision changed during the study time period (i.e. during the time period a city lost a provider)?

Please see the response to comment \#9.

Results:

19. The comparison between search engines is somewhat distracting and confusing. If it is significantly different in a consistent pattern, that is interesting. But it is hard to keep remembering facilitate versus not facilitate with anonymous different letters for the search engines. The authors may consider simplifying and removing the details of the differences between search engines and summarize as with the lines 217-221 in the discussion.

We have removed these results from the text in order to streamline the text and to make it clear that this was not a goal of our analysis.

20. It would be interesting to know if distance to a provider is also associated with region. One wonders if distance to a provider and region are really just confounding variables.

Distance to provider and region are indeed closely related (https://www.guttmacher.org/article/2017/10/disparities-and-change-over-time-distance-neededtravel-access-abortion-usa-spatial). The aim of our study was to gain insight into the experiences of individuals seeking abortion services in various cities throughout the country, and thus we did not account for any "confounding” variables.

Discussion:

21. Lines 221-25: Again, I wonder if distance to a provider and region are related.

Please see response to comment \#20.

22. Lines 243-259: while the anger is justified, this paragraph could be condensed with a focus on the internet aspects (could consider deleting the sentences on lines 246-7, 250-2).

We have condensed this paragraph, and it now reads as follows: 
Many results in our searches led to either crisis pregnancy centers or anti-abortion websites. Prior research has shown that crisis pregnancy centers intend to dissuade individuals from choosing abortion; they generally do not provide referrals for abortion care and are affiliated with anti-abortion organizations. ${ }^{16}$ One study of the websites of crisis pregnancy centers found that $80 \%$ provided incorrect information about abortion, ${ }^{17}$ and in our study, they were a common search result regardless of search terms or search engine. Another study reported that being unaware of a crisis pregnancy center's intended purpose can lead to surprise and anger about their refusal to provide or even refer for abortion care. ${ }^{13}$ Additionally, crisis pregnancy centers spend significant sums of money to advertise on internet search engines. ${ }^{16}$ Although Google banned such ads by religious organizations in 2008, a settlement was reached, and the ads are now allowed..$^{18}$ Google does have a policy against "misleading content," ${ }^{19}$ and in 2014, Google removed the ads of many crisis pregnancy centers. ${ }^{20}$ Despite this, we found that anti-abortion websites and crisis pregnancy centers were prominently featured among ads on all three search engines.

23. Lines 257: spell out NARAL

In order to not cite organizations that may be seen as political, we have edited this sentence to remove the name of the organization.

24. Lines 261-69: Adequately acknowledges the limitations of an internet study.

Thank you for this comment.

\section{STATISTICAL EDITOR’S COMMENTS}

25. Lines 123-124: Why were not more cities with smaller populations included? This strategy would seem to bias against more rural settings.

We had to limit the number of locations searched due to the time commitment of conducting the searches and classifying the results by hand. We chose the largest 25 cities to capture a substantial portion of the country's population. We chose the remaining state capitals in order to ensure that all states were included in the study. While this does exclude rural populations, we were unsure how to best search for rural locations when our possible locations were limited. Also, it is possible that individuals who live in rural settings search for services in larger cities nearby as opposed to in their small towns. Because abortion services are more likely to be located in urban settings, these findings are likely an optimistic estimate of the quality of information available online for abortion self-referral for the country as a whole. We have modified the end of the third paragraph of the Discussion as follows:

We found that searches in areas where the nearest abortion clinic was at least 100 miles away, which have recently been referred to as "abortion deserts, (Cartwright et al.), were the most likely to lead to inappropriate referrals; 27 U.S. cities, containing a total population in 2015 of over 3.3 million, are located within abortion deserts (Cartwright et al.). Thus, people who are most likely to rely on the internet are the least likely to access the information they need. Additionally, because abortion providers tend to be concentrated in more urban areas, which was the focus of this study, these findings likely provide an overestimate of the quality of information available online for abortion self-referral for the country as a whole. 
Additionally, we added the following sentence to the end of the limitations paragraph:

Finally, our search was limited to cities, and thus we were not able to characterize the quality of information available online for abortion self-referral in more rural areas.

26. Lines 159-210: While the figures are helpful, need to concisely state the important statistical comparisons in a Table format, so that the interested reader can see the differences in that format. Alternatively, could put the material in Figures in Table format with statistical comparisons and place that information as on-line supplemental.

We have included this information in three tables. They are currently designated as supplemental tables, though we are happy to have these included as main tables if preferred.

27. Should repeat in legend figures the 3 search engines used.

We have added the search engines to the figure legends.

\section{EDITOR'S COMMENTS}

28. Line 61: What was your analysis plan? Statistical methods? Descriptive only or comparisons

We have added a line to the end of the Methods stating that a chi-square test was used to make comparisons.

29. Line 71: If your intention is to do a comparison of information from webpages vs maps vs advertisements, please provide some statistical analysis to indicate what types of search results were most helpful vs least helpful.

We did not formally compare information obtained from webpages, maps, and ads. Instead, we present descriptive statistics of the proportion of each type of result that facilitated, did not facilitate, or hindered self-referral for abortion services. We added a conclusion to the Discussion that states that individuals seeking abortion services should be wary of the information found in ads, as they were the least likely to facilitate referral. If a formal statistical comparison would be helpful, we are happy to present pairwise comparisons between the proportion of each type of result that facilitated or hindered referral.

30. In the abstract, please provide absolute numbers as well as whichever effect size you are reporting + confidence intervals. P values may be omitted for space concerns. By absolute values, I mean something like xx (outcome in exposed) / yy (outcome in unexposed) (zz\%) (Effect size $=$; 95\% $\mathrm{CI}=$ ) An example might be: Outcome 1 was more common in the exposed than the unexposed $60 \% / 20 \%$ (Effect size=3; 95\% CI 2.6-3.4)

We are unclear what this comment is asking for in relation to our study. Is the editor referring to the results comparison by distance or Census region? If so, there are many results that could be included here. We will certainly provide this information if given more guidance on the specific results the editor would like included.

31. Line 76: Efforts by whom?

We have specified that these efforts were by a major search engine. 
32. Line 80: Common and legal?

We have added "and legal” to this sentence.

33. Line 99: Please clarify. Does the reference actually say that the providers said they would provide "misleading" information? If not, please edit.

We have removed "misleading” from this sentence.

34. Line 105: In discussion please consider acknowledging ACOG Committee Opinion, reaffirmed in 2016 entitled Number 385, November 2007 Reaffirmed 2016 Committee on Ethics The Limits of Conscientious Refusal in Reproductive Medicine in which the following statement appears: All health care providers must provide accurate and unbiased information so that patients can make informed decisions.

We have added the following line to the second paragraph of the Introduction:

Despite the American College of Obstetricians and Gynecologists' Committee Opinion stating that all health care providers must provide accurate and unbiased information so that patients can make informed decisions, ${ }^{6}$ individuals may not be able to obtain appropriate referrals from medical providers even after pregnancy diagnosis at a clinic visit.

We have also added the following sentence to the last paragraph of the Discussion:

As such, it is an ethical responsibility ${ }^{6}$ and critically important for health care providers and staff to be able to provide appropriate referrals for abortion care.

35. Line 106: Obtaining referrals through traditional methods?

We have modified this sentence to read "obtaining referrals through traditional methods.”

36. Line 115: It's not really the quality of referrals if the patient has sought information on the internet. I do agree with one of your reviewers who notes that the frame of reference for your report is that the patient is looking to get information about obtaining an abortion. It is likely that some women do internet searches on this topic with no intention to obtain an abortion, but rather looking specifically for crisis pregnancy centers. As such, the judgement that a particular internet search result assists or hinders obtaining accurate information is from that frame of reference. This needs to be explicitly stated in your paper.

We have modified the first sentence of the final paragraph of the Introduction to read as follows:

We sought to describe the quality of information available online for abortion self-referral in varying geographic regions in the United States assuming an individual was searching the internet to obtain information about where to obtain an abortion.

We also added the following sentence to the first paragraph of the Methods:

We designed these terms with the assumption that an individual using these terms would be searching specifically to locate abortion services and not searching for general information about abortion or for places to obtain other types of care. 
37. Line 115: Also, please describe your hypothesis if there is one and your primary and secondary (if any) objectives. Avoid using single sentence paragraphs.

We have revised the last paragraph of the Introduction to read as follows:

Our primary aim was to describe the quality of information available online for abortion selfreferral obtained from webpages, map results, and ads, assuming an individual was searching the internet to find information about where to obtain an abortion. Our secondary aim was to compare the quality of information obtained by geographic regions and distance to an abortion provider. We hypothesized that a substantial proportion of the results obtained when seeking information about where to obtain an abortion would not help an individual locate abortion care. We hypothesized also that geography and distance to an abortion provider would affect the proportion of results that helped an individual locate abortion care.

38. Line 116: It would seem that part of your objectives were to compare the search engines available.

Our rationale behind using three search engines was to capture most of the search engine market. We did not intend to compare the results between individual search engines, and we removed these comparisons from the manuscript text. We have modified a sentence in the third paragraph of the Methods to read as follows:

We report data as counts and proportions, and search engines are anonymized, as our objective was to describe the overall quality of information available online for abortion self-referral and not to compare individual search engines.

39. Line 123: For those less familiar with this capability, by turning location services off, how was the search engine able to identify the location of the IP address being used in order to locate local or nearby providers.

We have added the following to the first paragraph of the Methods:

We turned location services off, which prevents the computer from sending the latitude and longitude to the search engine; the search engine will use the city name included in the search and the location of the computer to geolocate the search.

We also edited the limitations paragraph of the Discussion to read as follows:

We prevented detailed location data from being accessed by the search engine, as we conducted all searches from one city. Our results could have been different had our searches originated from the cities for which we were searching due to the use of location identification by the search engines. However, tests of searches within one city distant from the study location showed nearly identical results when searching within that city and when using an anonymized location at the study site. While use of a virtual private network would have further prevented our study location from being used in the search results, this was not feasible.

40. Line 125: Perhaps just say this was a convenience sample.

We have revised the Methods to state that this was a sample of convenience.

41. Line 134: Please define what you mean by facilitated, neutral or hindering. This isn't clear.

The following information has been added to the third paragraph of the Methods: 
Data that facilitated abortion referral consisted of information that would theoretically allow the searcher to locate abortion services, while data that hindered abortion referral consisted of information that would potentially make it more difficult for the searcher to locate abortion services (e.g., a listing for an entity that does not provide referrals for the desired care). Data that did not facilitate abortion referral consisted of information that would be expected to have a neutral effect, i.e., it would not affect the searcher's ability to successfully locate abortion services.

42. Line 147: Please help with math: 68 cities 3 search engines=204 searches. Where do you get 612 searches?

We performed a total of 9 searches (3 search terms x 3 search engines) for 68 cities (25 most populous +43 state capitals that are not one of the prior 25 most populous cities); 9 x $68=612$. In order to make this easier for the reader to follow, we have modified the first sentence of the Results to read as follows:

We performed 612 searches using three search engines and three sets of search terms for 68 cities.

43. Line 157:Again: please help with math $68 \times 3 \times 10=2040$ webpages $\times 68$ cities $\times 3$ search engines $\mathrm{x} 10$ webpages. Do the same for each type of search.

Because each page of results could return fewer than 10 webpages, five map results, and five ads, this calculation is not straightforward. We have emphasized that each page could return fewer than the maximum number of results, and we have also corrected some errors in the number of results reported. The overall interpretation of the results did not change with these corrections.

44. Line 172: Please be consistent: did not facilitate or neutral?

We have ensured consistent use of the term “did not facilitate.”

45. Line 176: One reviewer requested that you identify the different search engines, I am fine with you keeping them anonymous.

Thank you. We have elected to keep them anonymous.

46. Line 185: Which type of searches? You have differentiated from webpages. Are these ads or map locations?

We have specified that this refers to map results.

47. Line 241: Please explain some where how search engine results are manipulated and how that could be region specific.

We don't believe that the search engine results are actually being manipulated. We are trying to link our finding of fewer search engine results for abortion providers in areas with fewer abortion providers to a prior finding that people are more likely to use the internet to search for abortion services when they live in areas with more restrictions on, and thus less access to, abortion. We have attempted to make this link clearer by modifying this section to now read as follows:

Individuals living in areas with multiple restrictions on abortion access, which tend to be areas with fewer abortion providers, are the most likely to use the internet to search for abortion. ${ }^{19}$ Such individuals are more likely to live farther from abortion clinics, as these restrictions have 
been associated with clinic closures. ${ }^{20}$ We found that searches in areas where the nearest abortion clinic was at least 100 miles away, which have recently been referred to as "abortion deserts, ${ }^{21}$ were the most likely to lead to inappropriate referrals; 27 U.S. cities, containing a total population in 2015 of over 3.3 million, are located within abortion deserts. ${ }^{21}$ Thus, people who are most likely to rely on the internet to locate abortion services are the least likely to access this information.

48. Line 246: Although this may be true, please temper this description as it will increase your credibility. As you write this, please consider how this would read to someone from a different perspective and then revise to be less inflammatory. You can say all of this without others claiming an enormous bias on your part and that will serve you well.

Please see response to comment \#22.

49. Line 263: Good place to emphasize how this is a single snap shot in time.

We have added the following sentence to the paragraph in the Discussion describing the study limitations:

Our study is also limited by its cross-sectional nature; while the top webpage results are expected to remain relatively stable, changes in the rankings of websites, map results, and ads do occur, and thus the results of this study represent a single snapshot in time. 

From:
To:
Randi Zung
Cc:
Subject:
Date:
RE: Your Revised Manuscript 18-1248R1
Attachments:
Wednesday, August 29, 2018 2:09:17 PM
18-1248R1 ms (8-24-18v2) Revised2.docx
Google AuthorDisclosure Neo.pdf

Hi Randi:

Please see below and attached documents.

Thank you,

Michele

1. Would you please resend the Author Agreement Form for Dr. Neo? Your previous email only contained one for Dr. Nipitta.

This is attached.

2. In Box 3 (Page 25), where should the asterisk and dagger symbols appear within the text? Please insert these missing footnote symbols.

These footnotes do not apply to Box 3, and we have removed them.

From: Randi Zung

Sent: Wednesday, August 29, 2018 1:28 PIVI

To: Hacker,Michele (HMFP - OB:GYN)

Cc: Dodge,Laura E. ( BIDMC - OB:GYN )

Subject: [External] RE: Your Revised Manuscript 18-1248R1

Dear Dr. Hacker:

Thank you for your responses. I have two follow-up queries.

1. Would you please resend the Author Agreement Form for Dr. Neo? Your previous email only contained one for Dr. Nipitta.

2. In Box 3 (Page 25), where should the asterisk and dagger symbols appear within the text? Please insert these missing footnote symbols.

The latest version of your manuscript (v3) is attached. Please send this back to me when you are finished.

Thank you,

Randi

From: Hacker, Michele (HMFP - OB:GYN)

Sent: Wednesday, August 29, 2018 12:01 PM 


\section{To: Randi Zung}

Cc: Dodge,Laura E. ( BIDMC - OB:GYN )

Subject: RE: Your Revised Manuscript 18-1248R1

\section{Dear Ms. Zung:}

Below are our responses to the queries. I have attached the Transparency Declaration, Dr. Nippita's disclosure and the revised manuscript.

Please let me know if you need anything additional.

Thank you, Michele

Michele R. Hacker, ScD, MSPH

Vice Chair for Research

Department of Obstetrics and Gynecology

Beth Israel Deaconess Medical Center

330 Brookline Avenue, Kirstein 3

Boston, MA 02215

Phone:

The information transmitted in this e-mail is intended only for the person or entity to which it is addressed. It may contain privileged or confidential material. Any review, retransmission, dissemination, or other use of this information by other than the intended recipient is prohibited. If you receive this e-mail in error, please contact the sender and delete the material from any computer.

1. General: The Editor has made edits to the manuscript using track changes. Please review them to make sure they are correct.

We have corrected the edits as needed, and the current edits are correct.

2. Please ask Dr. Neo to respond to their authorship confirmation email. We emailed them at . The email contains a link that needs to be clicked on. The sender of the email is EM@greenjournal.org.

This has been completed.

3. Please submit an Author Agreement form for Drs. Neo and Nippita with both the "Disclosure of Potential Conflicts of Interest" and "Authorship" sections completed. The form is available at http://edmgr.ovid.com/ong/accounts/agreementform.pdf. 
4. Our journal requires that all evidence-based research submissions be accompanied by a transparency declaration statement from the manuscript's lead author. The statement is as follows: "The lead author* affirms that this manuscript is an honest, accurate, and transparent account of the study being reported; that no important aspects of the study have been omitted; and that any discrepancies from the study as planned (and, if relevant, registered) have been explained." *The manuscript's guarantor.

Please provide a signed version of this statement.

This is attached.

5. Line 48: Please add the dates of the meeting.

We have added these dates.

6. Line 87: You haven't mentioned anything about this in the results or methods section. Is this true of all ads or just those related to family planning? Is this important to include in the conclusion of the abstract?

We don't know how helpful ads are in areas other than family planning. We agree that this is not important information to include in the abstract, and thus we have deleted this part of the sentence.

7. Line 88: In the results section, you just say that the results differed by region, but don't say how they differed. Thus, the conclusion statement that the quality is lowest in areas with least access is a black box-no data is given to support that. Also, you haven't told us "least access to abortion providers" is defined. This is important since its one of your major conclusions -also included in your precis. We need to know the definition of access.

We are referring to distance to an abortion provider as opposed to region, as we did not quantify abortion access in each region. In order to make this more clear to the reader, we have modified our final sentence of the abstract to read as follows:

Quality was lowest in areas that were farthest from abortion providers.

We have also modified a sentence in the second paragraph of the Discussion, which discusses the issue of distance to an abortion provider, access to an abortion provider, and quality of information available online, to read as follows:

Thus, people who are most likely to rely on the internet to locate abortion services are the least likely to access accurate information with which to locate an abortion provider.

8. Introduction: Your introduction and overall paper are a bit long. The introduction should be approximately 1 page long-yours is about 2.5. It is important to make the argument in the introduction about the relatively high rate of women not receiving any or accurate information from health care providers and that the internet is frequently the next (or first) choice but the level of detail could be decreased-perhaps some of it better in the discussion section?

We have shortened this section by removing some of the details.

9. Line 165: What do you mean by "up to". Why wouldn't you be consistent from one search to another? Perhaps the primary search strategy was based on using the first page only and then using all of the first page results, up to 10. Is that clearer and correct? Please make this edit.

We consistently categorized everything on the first page. We have revised this in an attempt to make this process clearer. 
10. Line 172: You need to make the statement that the primary outcome described in the intro (assess the quality of information) is the same as how you define it here. Ideally, you would describe these the same in both places. You could do this be stating your primary objective in the intro exactly as you have here on line 185-187. Then instead of repeating it here, just start with "Data that facilitated....." in the methods section.

We have moved this sentence to the Introduction and deleted it from the Methods.

11. Line 176: What about if the listing was for an entity that no longer exists? -ie, a dead URL?).

We did not click through all the links, and thus we do not know what proportion of links were inactive. However, we expect that few inactive links would be maintained on the first page of search results. We have added the following sentence to the limitations paragraph in the Discussion:

Additionally, we did not quantify the number of inactive links among the search results, though we expect that few inactive links were returned on the first page of results; we would not expect the proportion of inactive links to differ between organizations that facilitate, do not facilitate, and hinder abortion referral.

12. Line 183: Is this edit correct? It's important to be clear about your reference point for quality. Websites for pregnancy crisis centers could provide interactive, accurate, and easy to access information which some would argue is high quality.

Yes, this edit is correct.

13. Line 189: Please provide information about how you decided that there was an area abortion provider. For lowa, since telehealth options for medical abortion are available through Planned Parenthood, how did you characterize this?

We determined distance to an abortion provider by mapping the distance from the city to the closest publicly-listed (listed by the National Abortion Federation or Planned Parenthood or found on an internet search for abortion providers) abortion provider. The only city in lowa included in this study was Des Moines. There are abortion facilities within Des Moines, and thus this city is characterized as being $<50$ miles from an abortion provider. The provision of telemedicine in the rest of the state does not affect this classification.

14. Line 198: For data presented in the text, please provide the raw numbers as well as data such as percentages, effect size (OR, RR, etc) as appropriate and 95\% $\mathrm{Cl}^{\prime} \mathrm{s}$

We have added raw numbers throughout the Results.

15. Line 204: Please note the change in the previous sentence. Should this instance also be edited to say, "abortion provider"? Please make this edit if needed.

Yes, this should be "abortion provider." We have made this edit. We have also changed several edits of "health care provider" to "abortion provider" in cases where we are referring specifically to an abortion provider.

16. Box 1 (Page 20): Tables 2-4 are lists of information instead of data comparisons, so we'll present them as shaded boxes instead.

Thank you. 
17. Line 365 : Is there a definition missing for this text?

We have added two examples.

18 Line 382: Is there a definition missing for this text?

We do not have an example of this result.

19. Line 421: Please be sure the asterisk and dagger are noted in the box text.

We have ensured that this text is correct.

20. Line 440: Supplemental tables 1, 2, and 3 may be included in the print version of your manuscript. Please incorporate the numbering of these tables with your other tables and cite them in the text. Tables are cited in the order they are first mentioned. Cite each one as a "Table," not a "Supplemental Table."

We have changed the titles of these tables and have cited them in the text.

From: Randi Zung

Sent: Friday, August 24, 2018 9:46 AIVI

To: Hacker,Michele (HMFP - OB:GYN)

Subject: [External] Your Revised Manuscript 18-1248R1

Dear Dr. Hacker:

Your revised manuscript is being reviewed by the Editors. Before a final decision can be made, we need you to address the following queries. Please make the requested changes to the latest version of your manuscript that is attached to this email. Please track your changes and leave the ones made by the Editorial Office. Please also note your responses to the author queries in your email message back to me.

1. General: The Editor has made edits to the manuscript using track changes. Please review them to make sure they are correct.

2. Please ask Dr. Neo to respond to their authorship confirmation email. We emailed them at

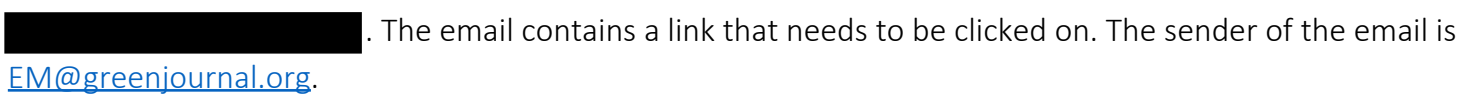

3. Please submit an Author Agreement form for Drs. Neo and Nippita with both the "Disclosure of Potential Conflicts of Interest" and "Authorship" sections completed. The form is available at

http://edmgr.ovid.com/ong/accounts/agreementform.pdf.

4. Our journal requires that all evidence-based research submissions be accompanied by a transparency declaration statement from the manuscript's lead author. The statement is as follows: "The lead author* affirms that this manuscript is an honest, accurate, and transparent account of the study being reported; that no important aspects of the study have been omitted; and that any discrepancies from the study as planned (and, if relevant, registered) have been explained." *The manuscript's guarantor.

Please provide a signed version of this statement. 
5. Line 48: Please add the dates of the meeting.

6. Line 87: You haven't mentioned anything about this in the results or methods section. Is this true of all ads or just those related to family planning? Is this important to include in the conclusion of the abstract?

7. Line 88: In the results section, you just say that the results differed by region, but don't say how they differed. Thus, the conclusion statement that the quality is lowest in areas with least access is a black box - no data is given to support that. Also, you haven't told us "least access to abortion providers" is defined. This is important since its one of your major conclusions -also included in your precis. We need to know the definition of access.

8. Introduction: Your introduction and overall paper are a bit long. The introduction should be approximately 1 page long-yours is about 2.5. It is important to make the argument in the introduction about the relatively high rate of women not receiving any or accurate information from health care providers and that the internet is frequently the next (or first) choice but the level of detail could be decreased-perhaps some of it better in the discussion section?

9. Line 165: What do you mean by "up to". Why wouldn't you be consistent from one search to another? Perhaps the primary search strategy was based on using the first page only and then using all of the first page results, up to 10. Is that clearer and correct? Please make this edit.

10. Line 172: You need to make the statement that the primary outcome described in the intro (assess the quality of information) is the same as how you define it here. Ideally, you would describe these the same in both places. You could do this be stating your primary objective in the intro exactly as you have here on line 185-187. Then instead of repeating it here, just start with "Data that facilitated....." in the methods section.

11. Line 176: What about if the listing was for an entity that no longer exists? -ie, a dead URL?).

12. Line 183: Is this edit correct? It's important to be clear about your reference point for quality. Websites for pregnancy crisis centers could provide interactive, accurate, and easy to access information which some would argue is high quality.

13. Line 189: Please provide information about how you decided that there was an area abortion provider. For lowa, since telehealth options for medical abortion are available through Planned Parenthood, how did you characterize this?

14. Line 198: For data presented in the text, please provide the raw numbers as well as data such as percentages, effect size (OR, RR, etc) as appropriate and 95\% $\mathrm{Cl}^{\prime} \mathrm{s}$

15. Line 204: Please note the change in the previous sentence. Should this instance also be edited to say, "abortion provider"? Please make this edit if needed.

16. Box 1 (Page 20): Tables 2-4 are lists of information instead of data comparisons, so we'll present them as shaded boxes instead.

17. Line 365 : Is there a definition missing for this text?

18 Line 382: Is there a definition missing for this text?

19. Line 421: Please be sure the asterisk and dagger are noted in the box text.

20. Line 440: Supplemental tables 1, 2, and 3 may be included in the print version of your manuscript. Please 
incorporate the numbering of these tables with your other tables and cite them in the text. Tables are cited in the order they are first mentioned. Cite each one as a "Table," not a "Supplemental Table."

To facilitate the review process, we would appreciate receiving a response by August 28.

Best,

Randi Zung

\section{Randi Zung (Ms.)}

Editorial Administrator | Obstetrics \& Gynecology

American College of Obstetricians and Gynecologists

409 12th Street, SW

Washington, DC 20024-2188

http://www.greenjournal.org

This message is intended for the use of the person(s) to whom it may be addressed. It may contain information that is privileged, confidential, or otherwise protected from disclosure under applicable law. If you are not the intended recipient, any dissemination, distribution, copying, or use of this information is prohibited. If you have received this message in error, please permanently delete it and immediately notify the sender. Thank you. 
Hi Stephanie:

The figures look good. We do not have any changes. Below is revised text for the legends to specify that distance is a stratification variable.

Please let us know if you need anything else.

Thank you,

Michele

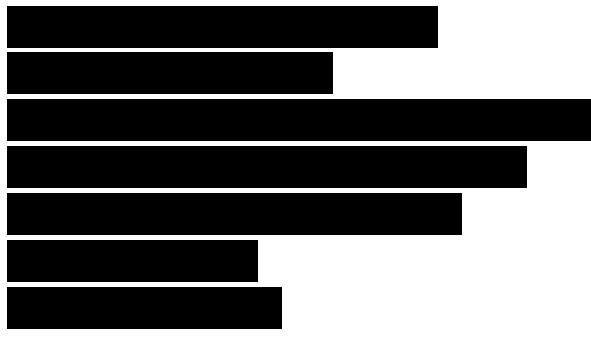

The information transmitted in this e-mail is intended only for the person or entity to which it is addressed. It may contain privileged or confidential material. Any review, retransmission, dissemination, or other use of this information by other than the intended recipient is prohibited. If you receive this e-mail in error, please contact the sender and delete the material from any computer.

Figure 1. The proportion of webpage results facilitating, hindering, and not facilitating referral stratified by anonymous search engine (Google, Bing, or Yahoo), search strategy (1 = “abortion” and location; 2 = "abortion clinic" and location; 3 = "where to get an abortion” and location), Census Bureau sub-region, and distance to an abortion provider

Figure 2. The proportion of location results facilitating, hindering, and not facilitating referral stratified by anonymous search engine (Google, Bing, or Yahoo), search strategy (1 = “abortion” and location; 2 = "abortion clinic" and location; 3 = "where to get an abortion” and location), Census Bureau sub-region, and distance to an abortion provider 
Figure 3. The proportion of ads facilitating, hindering, and not facilitating referral stratified by anonymous search engine (Google, Bing, or Yahoo), search strategy (1 = “abortion” and location; 2 = "abortion clinic” and location; 3 = "where to get an abortion" and location), Census Bureau sub-region, and distance to an abortion provider

From: Stephanie Casway [mailto:SCasway@greenjournal.org]

Sent: Wednesday, August 22, 2018 9:50 AM

To: Hacker, Michele (HMFP - OB:GYN)

Subject: [External] O\&G Art Revision: 18-1248

Good Morning Dr. Hacker,

Your figures and legend have been edited, and PDFs of the figures and legend are attached for your review. Please review the figures CAREFULLY for any mistakes.

PLEASE NOTE: Any changes to the figures must be made now. Changes at later stages are expensive and time-consuming and may result in the delay of your article's publication.

To avoid a delay, I would be grateful to receive a reply no later than Friday, 8/24. Thank you for your help.

Best wishes,

Stephanie Casway, MA

Production Editor

Obstetrics \& Gynecology

American College of Obstetricians and Gynecologists

409 12th St, SW

Washington, DC 20024

Ph: (202) 314-2339

Fax: (202) 479-0830

scasway@greenjournal.org

This message is intended for the use of the person(s) to whom it may be addressed. It may contain information that is privileged, confidential, or otherwise protected from disclosure under applicable law. If you are not the intended recipient, any dissemination, distribution, copying, or use of this information is prohibited. If you have received this message in error, please permanently delete it and immediately notify the sender. Thank you. 